\title{
Inhibition of STAT3-interacting protein 1 (STATIP1) promotes STAT3 transcriptional up-regulation and imatinib mesylate resistance in the chronic myeloid leukemia
}

\author{
André L Mencalha ${ }^{1,2,5^{*}+}$, Stephany Corrêa ${ }^{1 \dagger}$, Daniela Salles ${ }^{1,3}$, Bárbara Du Rocher ${ }^{1}$, Marcelo F Santiago ${ }^{4}$
} and Eliana Abdelhay ${ }^{1}$

\begin{abstract}
Background: Signal transducer and activator of transcription 3 (STAT3) is an important transcriptional factor frequently associated with the proliferation and survival of a large number of distinct cancer types. However, the signaling pathways and mechanisms that regulate STAT3 activation remain to be elucidated.

Methods: In this study we took advantage of existing cellular models for chronic myeloid leukemia resistance, western blot, in vitro signaling, real time PCR, flow cytometry approaches for cell cycle and apoptosis evaluation and siRNA assay in order to investigate the possible relationship between STATIP1, STAT3 and CML resistance.

Results: Here, we report the characterization of STAT3 protein regulation by STAT3-interacting protein (STATIP1) in the leukemia cell line K562, which demonstrates constitutive BCR-ABL TK activity. K562 cells exhibit high levels of phosphorylated STAT3 accumulated in the nucleus and enhanced BCR-ABL-dependent STAT3 transcriptional activity. Moreover, we demonstrate that STATIP1 is not involved in either BCR-ABL or STAT3 signaling but that STATIP1 is involved in the down-regulation of STAT3 transcription levels; STATIP1-depleted K562 cells display increased proliferation and increased levels of the anti-apoptosis STAT3 target genes CCND1 and BCL-XL, respectively. Furthermore, we demonstrated that Lucena, an Imatinib (IM)-resistant cell line, exhibits lower STATIP1 mRNA levels and undergoes apoptosis/cell cycle arrest in response to STAT3 inhibition together with IM treatment. We provide evidence that STATIP1 siRNA could confer therapy resistance in the K562 cells. Moreover, analysis of CML patients showed an inverse expression of STAIP1 and STAT3 mRNA levels, ratifying that IM-resistant patients present low STATIP1/high STAT3 mRNA levels.
\end{abstract}

Conclusions: Our data suggest that STATIP1 may be a negative regulator of STAT3 and demonstrate its involvement in IM therapy resistance in CML.

Keywords: STAT3, Chronic myeloid leukemia, BCR-ABL, STATIP1, Imatinib mesylate

\footnotetext{
* Correspondence: andre.mencalha@uerj.br

${ }^{\dagger}$ Equal contributors

'Bone Marrow Transplantation Unit (CEMO), National Cancer Institute (INCA),

Rio de Janeiro, Brazil

²Biophysics and Biometry Department, Roberto Alcântara Gomes Biology

Institute, Rio de Janeiro's State University (UERJ), Rio de Janeiro, Brazil

Full list of author information is available at the end of the article
} 


\section{Background}

The signal transducer and activator of transcription 3 (STAT3) protein belongs to a class of transcription factors that are activated by a number of growth factors and oncogenic proteins [1]. The activation of STAT3, which is regulated by the phosphorylation of tyrosine 705 , is driven by receptor and non-receptor protein tyrosine kinases (TK), such as EGFR, gp130, Ras, Src and Abl [2-5]. Once activated, STAT3 forms homodimers, translocate to the cell nucleus and binds to specific regulatory DNA elements to induce transcription. Under physiologic conditions, the activation of STAT3 is transient and rapid [6]. However, the persistent activation of STAT3 protein has been associated with several hematological cancers and solid tumors [7]. Previous data suggest that the constitutive activation of STAT3 induces cell transformation by the upregulation of anti-apoptotic and cell proliferation-related genes, such as $B C L-X L$ and CCND1 [7], and oncogenes, such as PIM1 and c-Myc [8,9]. Furthermore, STAT3 activation has been associated with the up-regulation of $V E G F$ and TWIST1, genes related to angiogenesis and metastasis [10]. These findings suggest a straight relationship between STAT3 activation and cancer development.

In chronic myeloid leukemia (CML), the chimeric oncoprotein $\mathrm{BCR}-\mathrm{ABL}$, a constitutively activated $\mathrm{TK}$, promotes the malignant transformation of hematopoietic cells [11]. BCR-ABL leads to the constitutive activation of the JAK/ STAT, Ras/Raf/MEK/ERK and PI3K/PTEN/Akt/mTOR signaling pathways [12-14]. In CML, persistent STAT3 phosphorylation mediated by BCR-ABL has been associated with cellular proliferation, the inhibition of apoptosis and chemotherapy resistance [5,15-19]. Although it is clear that the signaling activity of BCR-ABL is the main cause of the neoplastic transformation, the precise mechanisms by which BCR-ABL transforms cells remain largely unknown. Thus, strategies designed to understand the transcriptional activity of STAT3 may be important tools for discovering the next generation of anti-leukemia therapies.

STAT3 is negatively regulated by the suppressors of cytokine signaling proteins, known as SOCS, by protein inhibitor of activated STAT, known as PIAS, or by phosphatases, known as SHP. However, the regulatory mechanisms that negatively modulate STAT3 are ineffective in cancers [20]. Thus, several studies have tried to identify proteins that could interact and positively or negatively regulate STAT3 activity [21-28].

Although many proteins are known to interact and regulate STAT3 activity, the mechanisms surrounding such regulation of the STAT3 protein remain to be elucidated in CML. Collum and cols. [29] described STAT3interacting protein 1 (STATIP1) as a STAT3-associated protein. STATIP1 contains 12 WD40 domains that mediate protein-protein interactions, which play important roles in the regulation of signal transduction, transcription and proteolysis [30]. STATIP1 overexpression blocked STAT3 activation in the human hepatocellular carcinoma cell line HepG2 [29], suggesting a negative role for STATIP1 in STAT3 regulation. However, neither the STATIP1 expression nor its potential to regulate STAT3 activity has been assessed to date in other cancer types, such as leukemia cells. To address this issue, the aim of this study was to evaluate the STATIP1 and STAT3 status in the well-characterized CML model. Using K562 cell line, we report that STATIP1 may act as a negative regulator of STAT3 transcriptional activity in CML and reduce the effects of Imatinib (IM) in K562 cells. Moreover, using a CML multidrug resistance (MDR)/Imatinib resistant cell line (Lucena) and CML patients' samples we address the relationship of STATIP1 and STAT3 in IM resistance. Our results suggest a new role for STATIP1 in CML therapeutic resistance.

\section{Methods}

\section{Cell lines and drug treatments}

A CML model cell line, K562, was cultured in RPMI1640 medium containing 10\% fetal bovine serum, 100 $\mathrm{U} / \mathrm{ml}$ penicillin and $100 \mu \mathrm{g} / \mathrm{ml}$ streptomycin in $5 \% \mathrm{CO} 2$ at $37^{\circ} \mathrm{C}$. Lucena cells [K562 MDR/IM resistant cell line induced by vincristine] overexpressing $A B C B 1$ were kindly provided by Dra. Vivian Rumjanek (Departamento de Bioquímica Médica, Universidade Federal do Rio de Janeiro, Brazil) [31]. The Lucena cells were cultured in the same conditions as the $\mathrm{K} 562$ cells, but its medium was supplemented with $60 \mathrm{nM} \mathrm{VCR} \mathrm{(Sigma).The} \mathrm{K562} \mathrm{cells} \mathrm{were}$ plated at $1 \times 10^{5}$ cells $/ \mathrm{ml}$. The inhibition of BCR-ABL activity by treatment with IM (imatinib mesylate, Novartis) was performed using a final concentration of $1 \mu \mathrm{M}$ for $24 \mathrm{~h}$. For STAT3 inhibition, $40 \mu \mathrm{M}$ LLL-3 was applied to culture for $24 \mathrm{~h}$. The LLL-3 was kindly provided by Dr. Pui-Kai Li from Ohio State University, USA.

\section{Patients samples}

This study was approved by the ethics committee of the National Cancer Institute Hospital (INCA, Rio de Janeiro, Brazil). Patients were admitted or registered at the National Cancer Institute Hospital, according to the guidelines of its Ethics Committee and the Helsinki declaration. All patients and healthy donors were adults and signed the consent form. Bone marrow samples were obtained from CML patients in all disease phases (chronic, accelerated and blastic phases) at the time of diagnose and follow up: IM-responsive patients (3 to 6 mo follow up) and IM-resistant or relapse after initial response (3 to 24 mo follow up). We selected 6 healthy donors ( mean age $=30$, range $=20-37$, male:female ratio $=4: 2$ ), 6 IM-responsive patients (mean age $=45$, range $=35-68$, male:female ratio $=1: 5$ ) and 8 IM-resistant patients (mean 
age $=51$, range $=24-59$, male: female ratio $=6: 2$ ). Diagnoses and follow-ups were based on hematologic, cytogenetic and molecular assays. IM-responsive patients exhibited a major molecular response and complete hematologic and cytogenetic response, whereas IM-resistant patients lacked hematologic, cytogenetic and molecular responses. The inclusion criterion was to investigate CML patients that received IM as a first-line therapy. The exclusion criterion was CML patients with BCR-ABL mutations. Marrow aspirates were collected in heparinized tubes and processed on the day they were collected. Bone marrow mononuclear cells were isolated from $2-5 \mathrm{~mL}$ of aspirate in a FicollHypaque density gradient (Ficoll 1.077 g/mL; GE, Sweden) according to manufacturer's protocol. Cells were washed 3 times in PBS and subsequently used for RNA extraction.

\section{Small interfering RNA (siRNA)}

TK562 cells were plated at $1 \times 10^{5} \mathrm{cell} / \mathrm{ml}$ in a 24-well plate and left overnight in RPMI-1640 media without antibiotics. STATIP1 siRNA (100 nM) (SC-44436, Santa Cruz) and $2 \mu \mathrm{L}$ of Lipofectamine ${ }^{\mathrm{TM}}$ RNAiMAX (Invitrogen) were incubated separately in a final volume of $50 \mu \mathrm{L}$ of RPMI-1640 media for $5 \mathrm{~min}$. Subsequently, the siRNA and Lipofectamine were mixed and incubated for $30 \mathrm{~min}$ and then applied dropwise on cell cultures. Scrambled siRNA (100 nM) (SC-37007, Santa Cruz) was used as an siRNA negative control. FITC-conjugated siRNA (SC-36869, Santa Cruz) was used to evaluate the transfection efficiency by FACS. siRNA transfections were conducted for up to $72 \mathrm{~h}$.

\section{Proliferation assay}

K562 cells $\left(1 \times 10^{5}\right)$ were transfected with scrambled or STATIP1 siRNA in a 24-well plate for $72 \mathrm{~h}$. After transfection, cell cultures were treated with $1 \mu \mathrm{M}$ IM for $24 \mathrm{~h}$. WST-1 assay was performed to determine the number of viable cells. The relative number of viable cells was expressed as a percentage of the untreated cells.

\section{Real time quantitative PCR (RT-qPCR)}

Total RNA was extracted from IM-treated and untreated cells using TRIzol reagent (Invitrogen). Total RNA was subjected to treatment with a DNAse Amplification Grade I Kit (Invitrogen) for the removal of DNA contamination. Complementary DNA synthesis was performed with Superscript-II Reverse Transcriptase (Invitrogen) following the manufacturer's protocol. Quantitative RealTime PCR (RT-qPCR) was performed with SYBR Green Master Mix (Invitrogen) in a Rotor-Gene Q (Qiagen). The following forward (Fow) and reverse (Rev) primers were used: STAT3 - Fow 5' GGGAGAGAGTTACAGGTTGG ACAT 3', Rev 5' AGACGCCATTACAAGTGCCA 3'; STATIP1 - Fow 5' CCACTGTCCCTGCATTGGGATT 3', Rev 5' GCCACCTGCTGATACTCAAA 3'; CCND1- Fow
5’ AGAGACCAGGCTGTGTCCCTC 3', Rev 5' GTGGT GGCACGTAAGACACAC 3‘; BCL-XL Fow 5’ CTGGGG TCGCATTGTGGC 3', Rev 5' AGCCGCCGTTCTCCTG GA 3'; ABCB1 - Fow 5' CCCATCATTGCAATAGCAGG 3', Rev 5' GTTCAAACTTCTGCTCCTGA 3'; ACTB Fow 5' ACCTGAGAACTCCACTACCCT 3', Rev 5' GG TCCCACCCATGTTCCAG 3'. The PCR cycling conditions included an initial denaturation of $95^{\circ} \mathrm{C}$ for $10 \mathrm{mi}-$ nutes, followed by 45 cycles of 20 seconds at $95^{\circ} \mathrm{C}$, 20 seconds at $60^{\circ} \mathrm{C}$, and 40 seconds at $72^{\circ} \mathrm{C}$. The $\beta$-actin mRNA levels were used as a reference of expression. The fold-expression was calculated according to Schmittgen and Livak [32]. The primer sequences used in this work are available upon request.

\section{Western blot}

Whole-cell protein extracts were obtained from cell lines in lysis buffer containing $50 \mathrm{mM}$ Tris $\mathrm{pH}$ 7.5, $5 \mathrm{mM}$ EDTA, $10 \mathrm{mM}$ EGTA, $50 \mathrm{mM}$ NaF, $20 \mathrm{mM}$ b-glycerolphosphate, $250 \mathrm{mM} \mathrm{NaCl}, 0.1 \%$ Triton $\mathrm{X}-100,20 \mathrm{mM} \mathrm{Na}_{3} \mathrm{VO}_{4}$ and protease inhibitor mix (Amersham). The protein concentrations were determined using the Bradford assay, and $30 \mu \mathrm{g}$ of the cell lysate proteins was subjected to separation by $10 \%$ SDS-PAGE. The protein extracts were electrophoretically transferred to a nitrocellulose membrane (GE) and probed with the appropriate antibodies. The western blots were developed by ECL Plus (Amersham). The following antibodies were used at 1:1000 dilutions: antiSTATIP1, anti-STAT3, anti-STAT3-Y705 and antiACTNB (Santa Cruz).

\section{Immunofluorescence}

K562 cells were fixed to glass slides using cytospin and further fixed by immersion in methanol:acetic acid (1:1) for $10 \mathrm{~min}$ at $-20^{\circ} \mathrm{C}$. Fixed cells were permeabilized in $0.5 \%$ Triton X-100 for 10 minutes and blocked with $5 \%$ BSA for $1 \mathrm{~h}$. Primary antibody incubation was performed at $4^{\circ} \mathrm{C}$ for $16 \mathrm{~h}$. The cell nuclei were stained with DAPI (Santa Cruz). The images were analyzed using a LSM 510 Meta (Carl Zeiss) microscope equipped with a $63 \times 1$ 1.4 NA Plan-Apochromat oil immersion objective.

\section{Apoptosis assay}

To determine the percentage of apoptotic cells, we analyzed phosphatidyl serine externalization and membrane integrity by double staining with Annexin V PE and 7-AAD (PE Annexin V Apoptosis Detection Kit I, BD Pharmingen, USA) according to manufacturer's instructions. Briefly, after treatment, $1.0 \times 10^{5}$ cells were harvested, washed twice with cold PBS and resuspended in $100 \mu \mathrm{L}$ of $1 \times$ binding buffer. Annexin V PE $(5 \mu \mathrm{L})$ and 7 -AAD $(5 \mu \mathrm{L})$ were added, and samples were incubated for $15 \mathrm{~min}$ in the dark. After incubation, $400 \mu \mathrm{L}$ of $1 \mathrm{X}$ binding buffer was added to each sample. Cells positive for Annexin V PE and 7-AAD were 
considered apoptotic. For every condition, 20.000 events were acquired using a FACSCalibur Flow Cytometer (Becton Dickinson, USA) and analyzed using CellQuest v.3.1 Software (Becton Dickinson, USA). All experiments were performed in triplicate.

\section{Cell cycle assays}

Cell cycle was evaluated by staining with propidium iodide (PI, Sigma-Aldrich) [33]. Approximately $3.0 \times 10^{5}$ cells were resuspended in $400 \mu \mathrm{L}$ of hypotonic buffer (3.4 mM Tris- $\mathrm{HCl}$ ( $\mathrm{pH}$ 7.6), $10 \mathrm{mM} \mathrm{NaCl}, 0.1 \%$ (v/v) NP-40, $700 \mathrm{U} / \mathrm{L}$ RNase, and $0.075 \mathrm{mM} \mathrm{PI})$ and incubated for $30 \mathrm{~min}$ at $4^{\circ} \mathrm{C}$. For every condition, 5.000 events were acquired in a FACSCalibur Flow Cytometer (Becton Dickinson, USA) and analyzed using Cell Quest v.3.1 Software (Becton Dickinson, USA). All experiments were performed in triplicate.

\section{Statistical analysis}

All of the experiments were repeated at least three times, and the data are expressed as the mean \pm SD. Statistical analyses (ANOVA and t-test) were performed using GraphPad Prism ${ }^{\odot}$ v.5 software (GraphPad). A $\mathrm{P}$-value $(\mathrm{p})<0.05$ was considered statistically significant $\left({ }^{*} \mathrm{p}<0.05, * * \mathrm{p}<0.01\right.$, *** $\left.\mathrm{p}<0.001\right)$.

\section{Results}

\section{Evaluation of STAT3 expression and phosphorylation in CML K562 cells}

Previous studies have demonstrated that STAT3 is constitutively activated in a variety of cancer cell types [7], including leukemic cells [34]. First, we evaluated the STAT3 expression and phosphorylation status and subcellular localization in our CML cell line, K562. For this, immunofluorescence assays and western blot analyses were performed. Our results indicate that STAT3 is preferentially localized in the $\mathrm{K} 562$ cytoplasm, while a very strong nuclear accumulation of phosphorylated STAT3 is observed in these cells (Figure 1F, 1I). These findings indicate that when STAT3 is phosphorylated, it accumulates in the K562 cell nucleus. These data validate our model as a STAT3-activated leukemic cell line, as reported by Benekli and cols. [7], who described STAT3 phosphorylation as a common finding in leukemic and other cancer cells.

Inhibition of BCR-ABL interferes with STAT3 modifications but does not alter STATIP1 protein expression

To demonstrate the role of BCR-ABL in STAT3 phosphorylation and the possible consequence of this signaling on STATIP1 expression, we first investigated the status of STAT3 and STATIP1 expression and STAT3 tyrosine-705 phosphorylation in BCR-ABL-inhibited K562 cells by immunofluorescence assays and western blotting. We inhibited BCR-ABL activity with $1 \mu \mathrm{M}$ IM (Figure 1J-R), as previously described [35]. Although BCR-ABL coordinates several molecular alterations, the STATIP1 protein levels remained unaltered following BCR-ABL inhibition using $1 \mu \mathrm{M}$ IM for $24 \mathrm{~h}$ (Figure $1 \mathrm{C}, 1 \mathrm{~L}$ ). However, the STAT3 protein levels, phosphorylation status and nuclear accumulation were decreased in IM-treated cells compared with non-treated K562 cells (Figures 1R and 2A, C-D). Unlike STAT3, our data suggested that STATIP1 expression is not related to BCR-ABL signaling (Figures 1L and 2A, C-D).

\section{Imatinib treatment induces down-regulation of STAT3 target} genes but not alteration of STATIP1 transcript levels

Several genes listed as STAT3 targets exhibit a relevant role in cancer [7-10]. STAT3 target genes mainly include cellular growth promoters and inhibitors of apoptosis [36]. Moreover, STAT3 has been described as an activator of its own transcription [37]. Here, we investigated the regulation of STAT3 target genes in K562 cells in response to IM treatment. The mRNA levels of CCND1, $B C L-X L$ and STAT3 genes were measured by RT-qPCR. Our results suggest that STAT3 target genes were downregulated $24 \mathrm{~h}$ after IM treatment (Figure 2A). To assess the direct activity of STAT3 on its gene targets, we directly inhibited STAT3 using LLL-3. In corroboration with the previous results, the CCND1, BCL-XL and STAT3 mRNA levels were down-regulated in K562 cells after $24 \mathrm{~h}$ with LLL-3 treatment compared to untreated cells (Figure 2B). These findings indicate that STAT3 inhibition either indirectly, by IM, or directly, by LLL-3, induces a decrease in STAT3 transcriptional activity. Additionally, STAT3 inhibition with LLL-3 also does not interfere with the STATIP1 mRNA levels (Figure 2B). Our data indicated that STATIP1 is not correlated with either the BCR-ABL or STAT3 signaling pathways but that it may be related to STAT3 activity in the CML cell line.

\section{STATIP1 depletion results in increased STAT3 transcriptional activity in K562 cells}

Previous studies have demonstrated that STAT3 activity can be regulated by STAT3 protein interactions $[23,27,38]$. To determine the potential of STATIP1 in regulating the transcriptional activity of STAT3, K562 cells were transfected with siRNA against STATIP1. The mRNA levels were analyzed and compared to untransfected or scrambled-transfected K562 cells. By RT-qPCR, significant decreases in the STATIP1 mRNA and protein levels were observed $72 \mathrm{~h}$ after siRNA transfection (Figure 3A,B). Interestingly, the increase in STAT3 mRNA levels after STATIP1 inhibition were inversely proportional, showing significant elevation at $72 \mathrm{~h}$ (Figure $3 \mathrm{C}$ ). This result suggests that with transient STATIP1 depletion, STAT3 is more transcriptionally 


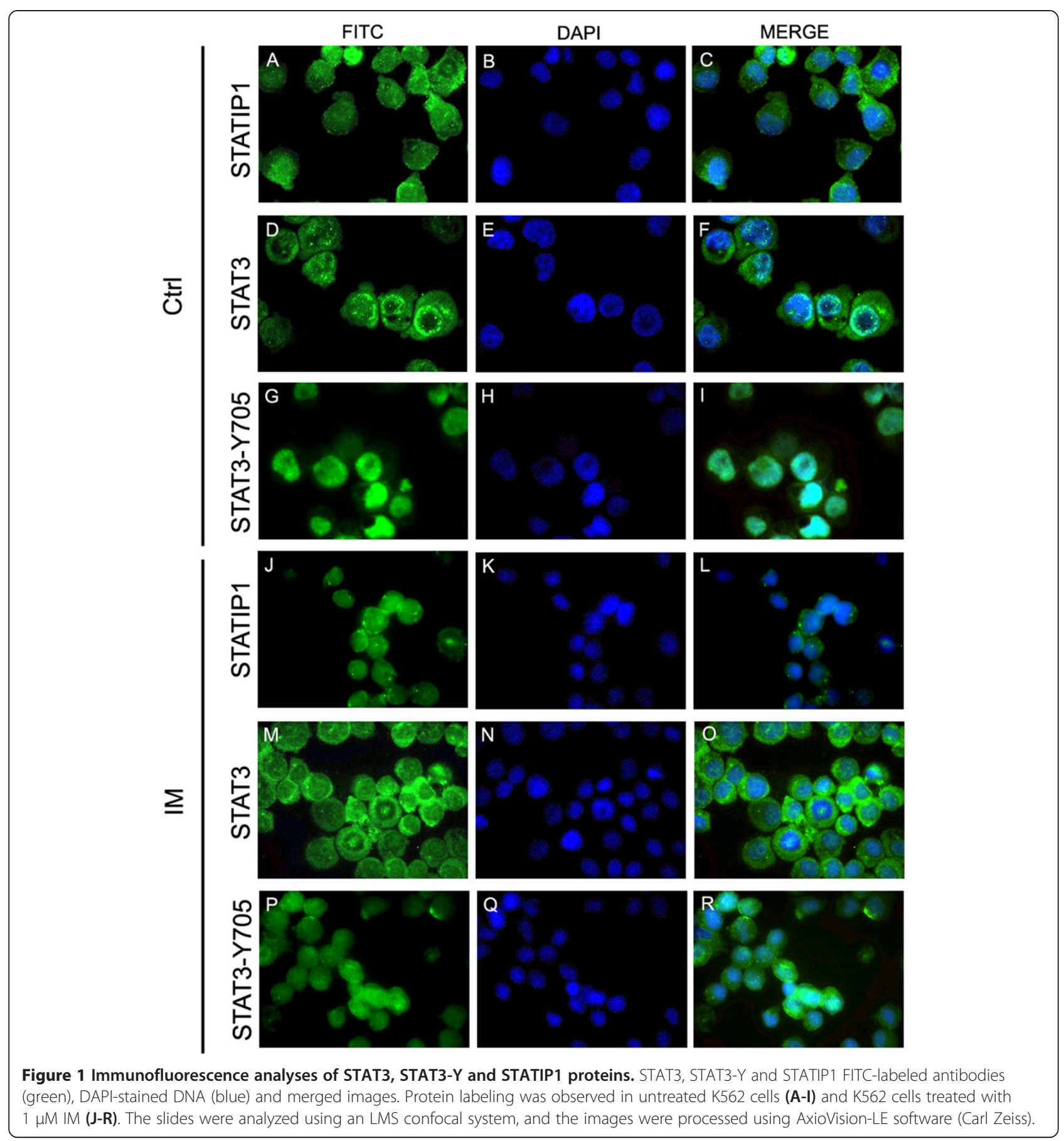

activated. To validate this hypothesis, we investigated STAT3 target gene mRNA levels. Surprisingly, in response to STATIP1 inhibition, a significant two-fold increase of CCND1 mRNA levels and a three-fold increase of $B C L-X L$ mRNA levels were observed $72 \mathrm{~h}$ after siRNA transfection (Figure 3D). These findings showed that STATIP1 down-regulation in K562 cells augments the STAT3 mRNA levels and its targeted genes, demonstrating that STATIP1 is involved (directly or indirectly) in the negative regulation of STAT3 transcription.

\section{STATIP1 is involved in imatinib resistance in CML}

The role of STATIP1 both physiologically and in cancer cells is completely unknown. In an effort to determine the mechanism of STATIP1-mediated CML therapy resistance, we used the Lucena cell line as a model of IM 


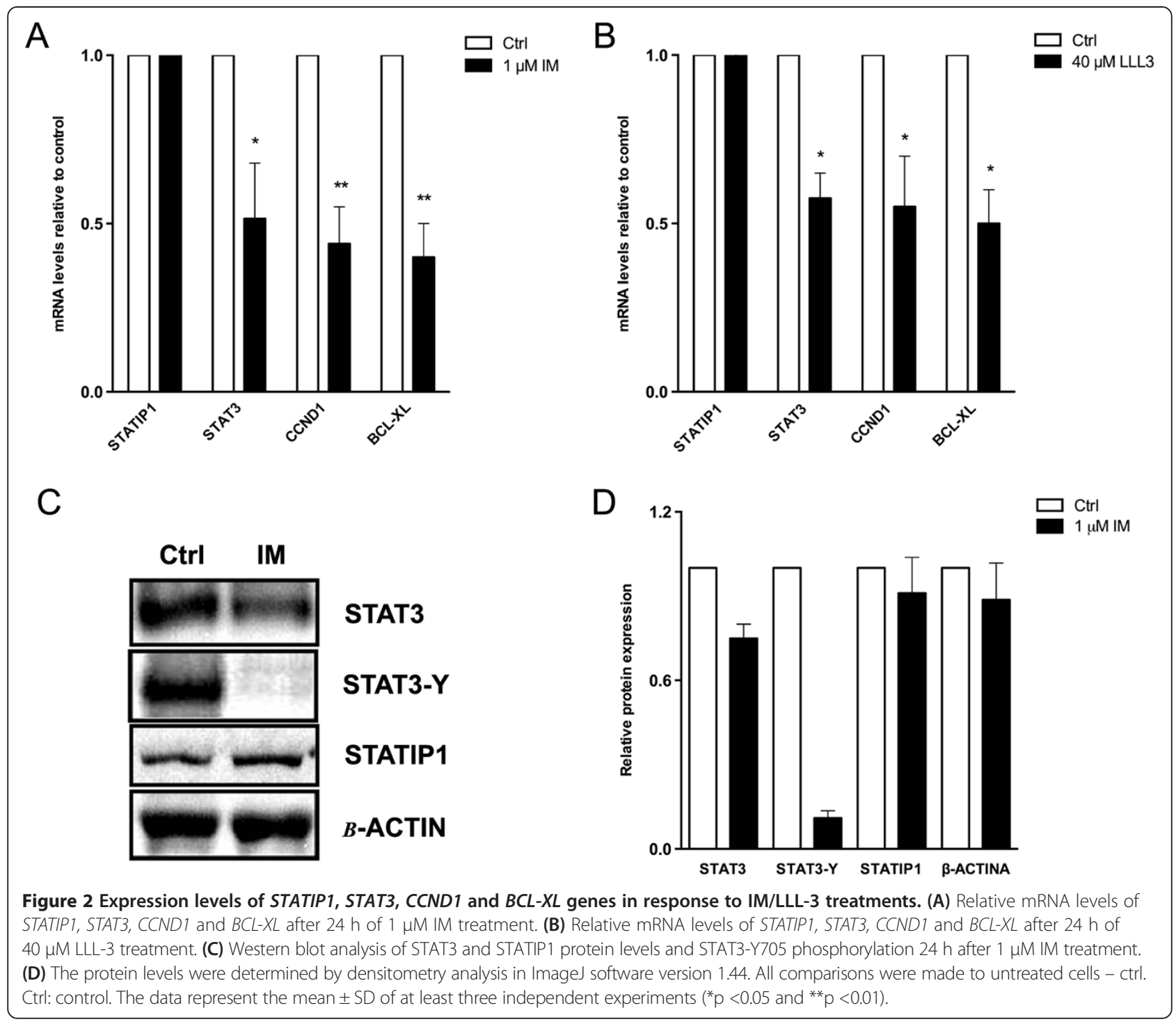

resistance [39]. Lucena cells were subjected to IM, LLL3 , and co-treatment (as previously reported) [35], and the STAT3, STATIP1 and ABCB1 mRNA levels were evaluated after $24 \mathrm{~h}$ and compared to untreated cells. The STATIP1 mRNA levels were lower in the Lucena cells compared to untreated K562 cells (Figure 4A). Additionally, the STAT3 mRNA levels decreased by $60 \%$ in the Lucena cells with each of the different treatments (Figure 4B), but the $A B C B 1 \mathrm{mRNA}$ levels only decreased with the LLL-3 treatment ( $\cong 50 \%$ ) (Figure $4 C$ ). No differences were observed regarding the $A B C B 1$ mRNA levels in K562 cells (data not shown). Interestingly, STAT3 inhibition by LLL-3 treatment sensitized Lucena cells to IM treatment (Figure 4D) in a cell cycle arrest-independent manner (Figure 4E-F). Together, these results suggest that IM resistance may be associated not only with STAT3 overexpression/activation but also with STATIP1 downregulation.
To address this hypothesis, we assessed K562 cell viability after IM treatment in STATIP1-depleted cells $72 \mathrm{~h}$ after siRNA transfection. Our results indicated a decrease in the IM sensitivity of the $\mathrm{K} 562$ cell line with reduced STATIP1 expression compared to the control or scrambled K562 cells (Figure 5). After 24 h of $1 \mu \mathrm{M}$ IM treatment, approximately $25 \%$ of the STATIP1-depleted K562 cells remained viable compared to the control or scrambled cells (Figure 5). Additionally, we also analyzed a total of $14 \mathrm{CML}$ patients with different responses to IM (6 IM-responsive and 8 IM-resistant) and 6 healthy bone marrow donors. RT-qPCR analyses showed that IM-resistant patients presented STATIP1 mRNAs levels down-regulated, compared to IM-responsive patients (Figure 6A). Moreover, STAT3 mRNA levels were inversely expressed; up-reguleted in IM-resistant patients, compared to IM-responsive (Figure 6B). These data suggest that the decreased expression of STATIP1 may 

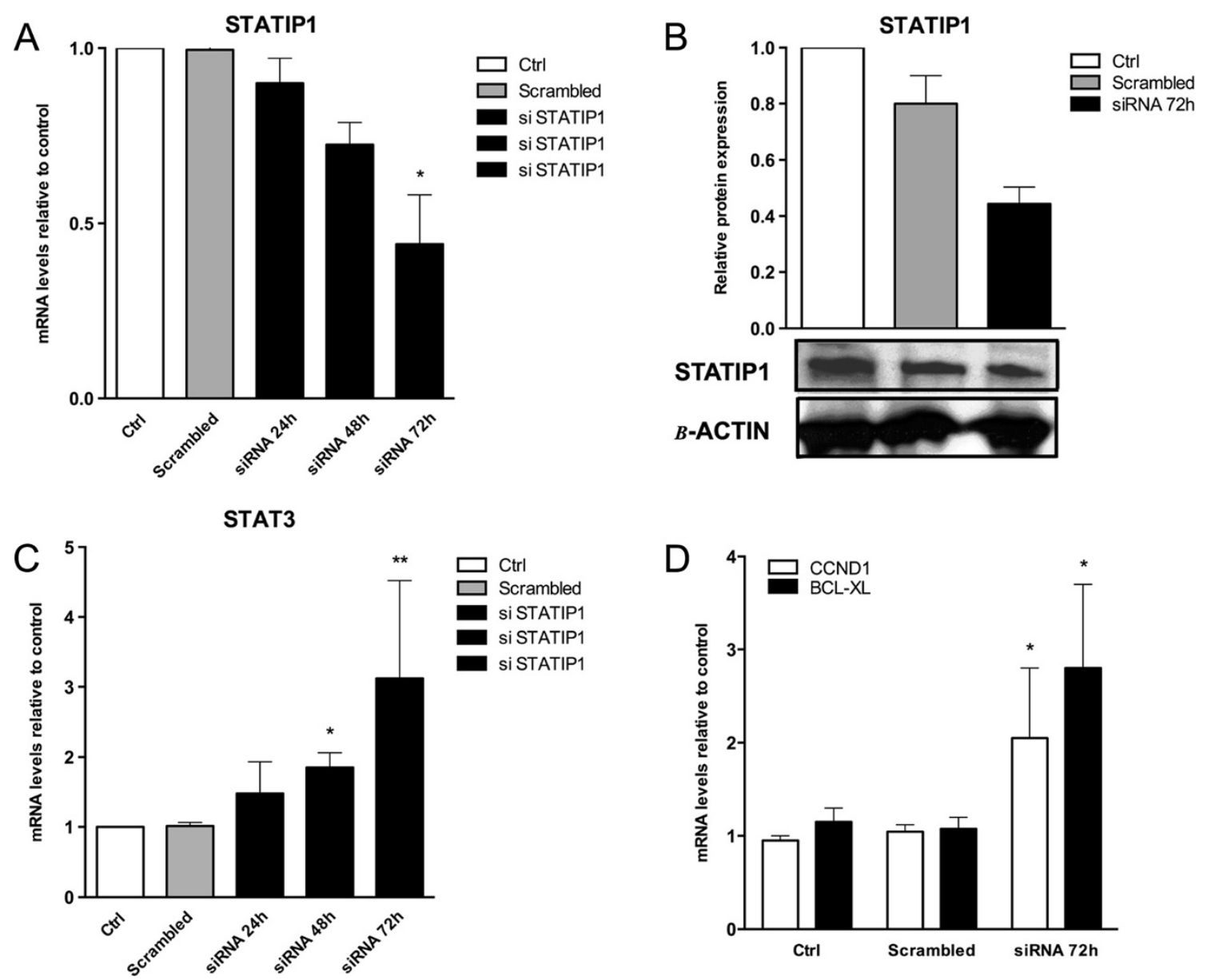

Figure 3 STATIP1 mRNA depletion by siRNA induces the over-expression of STAT3 and its target genes. (A) STATIP1 mRNA levels at $24 \mathrm{~h}$, $48 \mathrm{~h}$ and $72 \mathrm{~h}$ after STATIP1 silencing, as determined by RT-qPCR. (B) Western blot analyses of the STATIP1 protein level $72 \mathrm{~h}$ after STATIP1 silencing. (C) RT-qPCR analyses of the STAT3 mRNA levels at $24 \mathrm{~h}, 48 \mathrm{~h}$ and $72 \mathrm{~h}$ after STATIP1 silencing. (D) RT-qPCR analyses of the CCND1 and $B C L-X L$ mRNA levels at $72 \mathrm{~h}$ after STATIP1 silencing. All comparisons were made to untreated cells $-\mathrm{ctrl}$ and scrambled-treated cells. Ctrl: control. The data represent the means \pm SD of at least three independent experiments $\left({ }^{*} p<0.05\right.$ and ${ }^{* *} p<0.01$ ).

promote IM resistance in the K562 cell line, and could be an important piece of in vivo IM-resistance development in CML.

\section{Discussion}

Although the BCR-ABL oncoprotein, a hallmark of CML, constitutively activates multiple signaling pathways [3], our group was particular interested in STAT3 signaling activation, as the constitutive activation of STAT3 is associated with oncogenic transformation induced by the viral Src oncoprotein [2]. Furthermore, many in vivo and in vitro assays have demonstrated the association of STAT3 activation with the development and maintenance of several cancer types $[1,4,10,16]$. Despite the known association of STAT3 phosphorylation with cancer, the mechanisms that regulate STAT3 are not well understood.
In this study, we were able to clarify the relationship between BCR-ABL signaling and STAT3 activation. Our data indicated strong STAT3 phosphorylation and nuclear accumulation in untreated K562 cells. K562 treatment with IM, an inhibitor of BCR-ABL activity, not only promoted a decrease in the mRNA and protein levels of STAT3 but also inhibited STAT3 phosphorylation. Moreover, our results also showed a transcriptional positive feedback loop, suggesting that STAT3 promotes its own over-expression, which may be important to signaling intensification. In summary, our findings suggest that STAT3 is phosphorylated and transcriptionally activated by BCR-ABL activity in K562 cells.

Several studies have demonstrated that STAT3 signaling can regulate the expression of numerous genes that are frequently involved with proliferation and apoptosis [34], angiogenesis, metastasis and differentiation [36], some of which are capable of positively regulating STAT3 through 


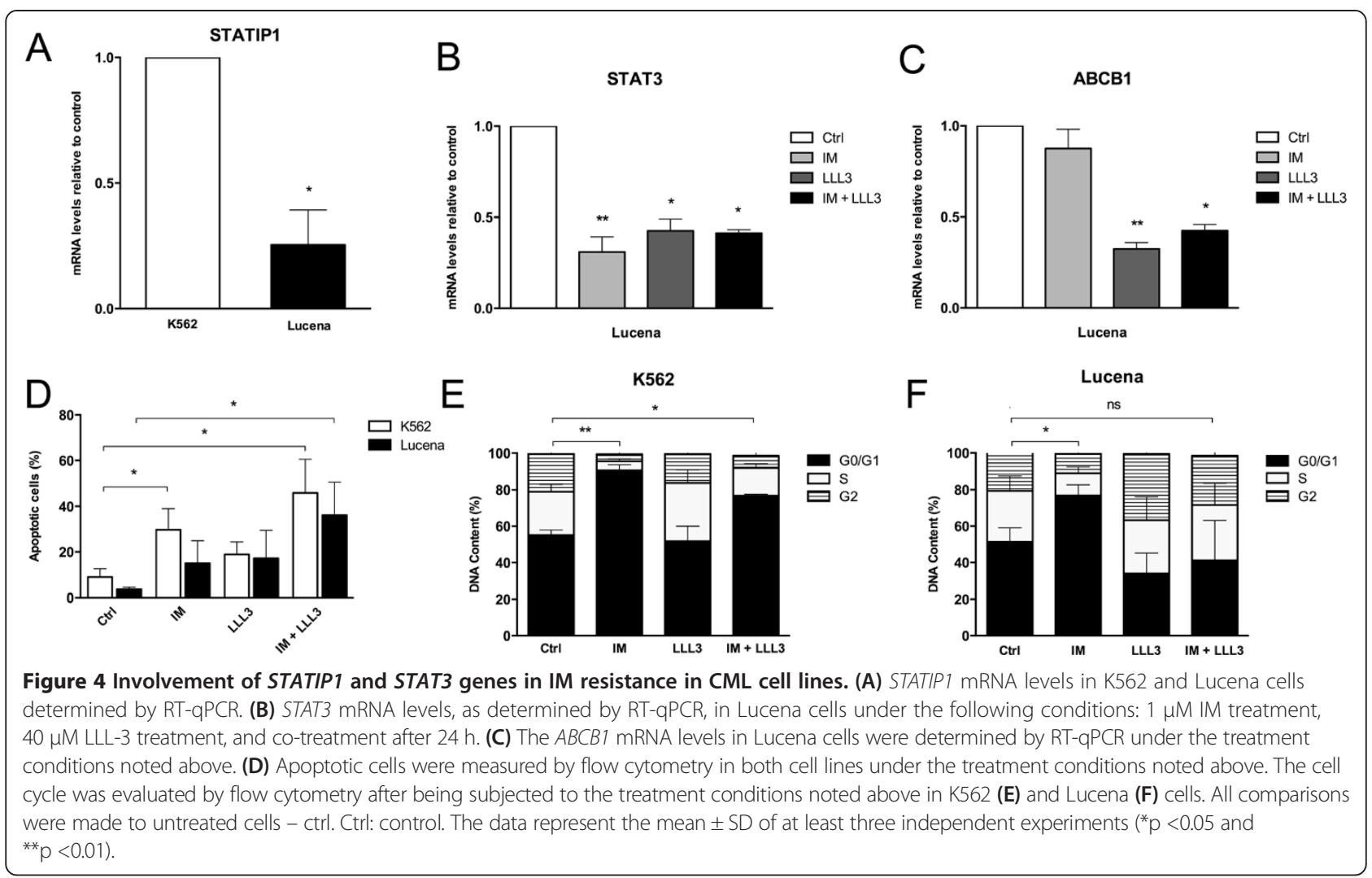

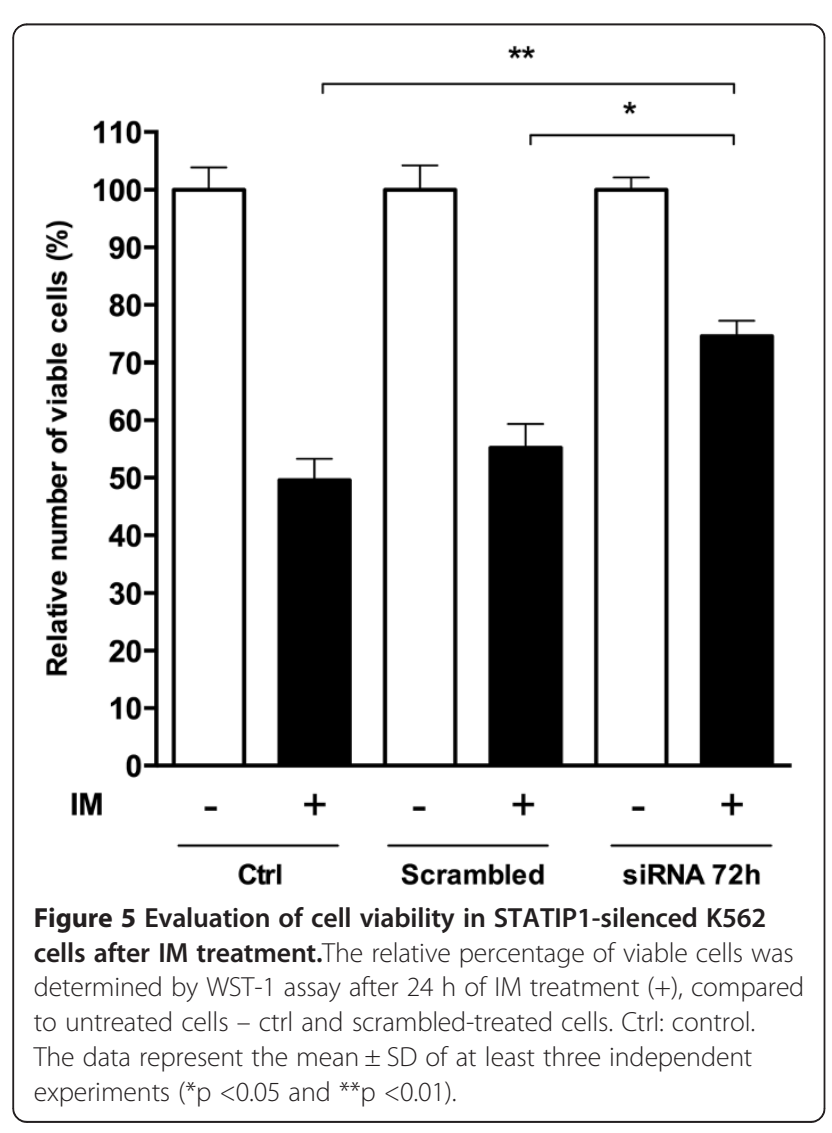

protein-protein interactions. To probe the diminished STAT3 activation in BCR-ABL-inhibited cells, we assessed the expression of representative known STAT3 target genes involved in proliferation and cellular survival, CCND1 and $B C L-X L$, respectively, and STATIP1, a protein identified in a two-hybrid assay as interacting with STAT3 [29]. As expected, CCND1 and BCL-XL were down-regulated in response to IM treatment, but unlike STAT3, the STATIP1 mRNA and protein levels were unaltered in the treated cells. Accordingly, our results indicated that STATIP1 was not affected by the molecular alterations promoted by BCR-ABL signaling.

Hawkes and cols. characterized the STATIP1 levels in the cytoplasm and nuclei of cancer cell lines exercising multiple distinct roles that are dependent on its subcellular localization [40]. To further investigate the relationship between STAT3 and STATIP1 in the context of BCR-ABL, we inhibited STAT3 activity with LLL-3, a more direct approach that has been previously used by our group [35]. Similar to the BCR-ABL inhibition experiments, previously investigated STAT3 target genes demonstrated decreased mRNA levels compared to untreated cells. STATIP1 remained unchanged in K562 cells treated with the STAT3 drug inhibitor LLL-3. This result corroborates our previous results, again suggesting that STATIP1 expression is not related to molecular signaling changes driven by either BCR-ABL or STAT3. Moreover, our 

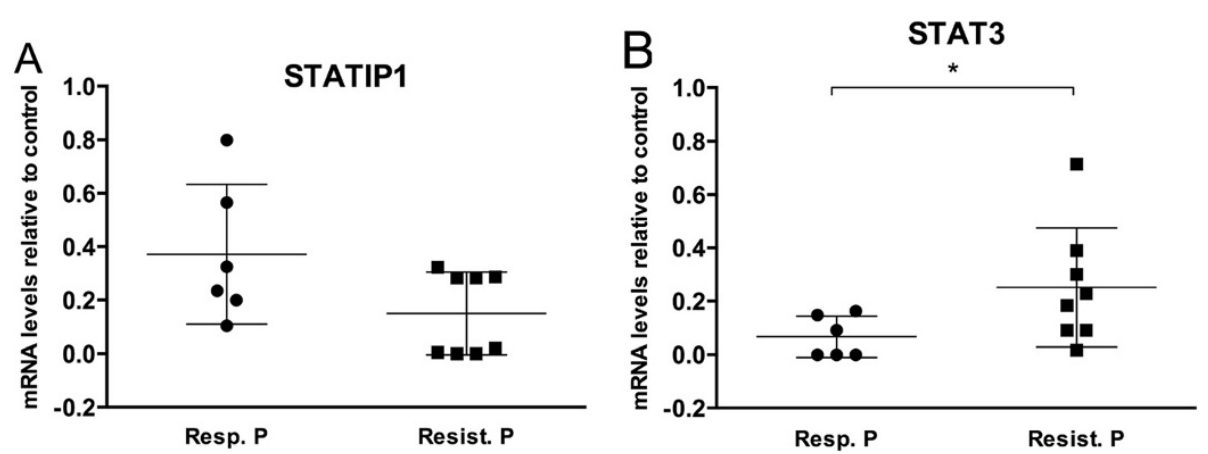

Figure 6 Expression levels of STATIP1 and STAT3 genes in CML patients. (A) STATIP1 mRNA levels and (B) STAT3 mRNA levels were determined by RT-qPCR analyses in 6 IM-responsive patients and 8 IM-resistant patients. Raw expression values were normalized to $\beta$-actin expression. Expression changes were calibrated by 6 healthy bone marrow donors analysis. Resp. $P=$ responsive patients; Resist. P. $=$ resistant patients. $\left({ }^{*} p<0.05\right)$.

findings showed that STATIP1 is present in both the cytoplasm and nuclei of K562 cells. Further characterization of the localized STATIP1 pools could reveal its precise role in these cellular compartments.

It is known that STATIP1 contains 12 WD40 domains that are responsible for mediating protein-protein interactions that play important roles in signal transduction regulation, transcription and proteolysis [30]. In this context, the investigation of the role of STATIP1 in signal transduction showed that its forced over-expression is able to block STAT3 activation [29]. However, the regulation of STAT3 transcriptional activity by STATIP1 was only observed in the human hepatocellular carcinoma cell line HepG2 [29]. In this study, we characterized STATIP1 in the K562 cell line and investigated its role in STAT3 transcriptional activity in a distinct cell line established from another cancer type, chronic myeloid leukemia. Instead of over-expressing STATIP1, as was performed by Collun and cols. [29], we depleted the STATIP1 mRNA and protein levels to investigate the role of STATIP1 in regulating STAT3 transcriptional activity in K562 cells. Our results showed a gradual increase of STAT3-target gene mRNA levels, such as those of STAT3, CCND1 and BCL-XL, in K562 cells subjected to STATIP1 inhibition. Similarly to Collun [29], our findings also indicated that STATIP1 may work as a negative regulator of STAT3 transcriptional activity. Because STATIP1 interacts with STAT3, we inferred that this may be a direct regulation mechanism. Indeed, existing data have already characterized STATIP1 protein as a scaffolding protein that regulates the activity of interacting proteins [40]. Based on this finding, we propose that STATIP1 may interact with STAT3 in K562 cells and regulate STAT3 activation. However, additional investigation is required to address the intricate mechanism by which STAT3 is inhibited by STATIP1. Nevertheless, independent of whether it is a direct or indirect regulation and how it works precisely, our results demonstrated that negative regulation of STAT3 by STATIP1 appears to be a common issue in distinct cancer cell types. If this result is validated in other diverse cancer cell types, we propose that STAT3 regulation may be important to cancer development and that it may also be an interesting target for the design of new drug strategies against cancer cells.

Because STAT3 over-expression is closely related to CML drug resistance and has been implicated in a poor prognosis $[17,41]$, we evaluated the role of STATIP1 in IM resistance. We took advantage of an IM-resistant cell model, the Lucena cell line. Lucena cells exhibit a multidrug resistance phenotype (with $A B C B 1$ over-expression) and have been shown to also be IM resistant, compared to K562 cells [39]. We investigated the STATIP1, STAT3, and $A B C B 1$ mRNA levels, together with apoptosis and cell cycle arrest, in Lucena cells with the inhibition of BCRABL and STAT3.

We observed decreased STATIP1 mRNA levels in Lucena cells compared to K562 cells. Because Lucena cells are resistant to IM, we observed STAT3 down-regulation in all of the treatments; additionally, we observed a decrease in the $A B C B 1$ mRNA levels. This result was expected because it is known that $A B C B 1$ is a STAT3 target $[42,43]$. Moreover, STAT3 direct inhibition (LLL-3 treatment) induced Lucena cells to undergo apoptosis, in contrast to indirect inhibition (IM treatment), and this effect was independent of cell cycle arrest. This result demonstrated that STAT3 over-expression together with STATIP1 down-regulation could be involved in IM resistance.

To validate this hypothesis, we depleted STATIP1 and inhibited BCR-ABL activity in K562 cells and assessed the proliferation and survival. Interestingly, our results demonstrated that STATIP1-depleted K562 cells have a higher survival percentage than control or scrambled-transfected cells. STAT3 can overcome sensitivity to BCR-ABL inhibition by driving proliferation, anti-apoptosis and MDR gene expression, increasing CML cell survival [15-19,43]. 
Moreover, althought we analyzed a small cohort of healthy donors and patients samples, our in vivo analyses suggested that STAT3 and STATIP1 genes are inversely expressed in IM-response, which corresponds to our findings in K562 and Lucena cell lines. The present study is the first report of STATIP1 expression in CML patients with different responses to IM therapy. Further studies may reveal the details of STATIP1 role in IM resistance.

\section{Conclusions}

Our data suggest that STATIP1 may be a negative regulator of STAT3 and that it could be involved in the acquisition of therapeutic resistance to IM in CML.

\section{Abbreviations \\ CML: Chronic myeloid leukemia; Fow: Forward; IM: Imatinib mesylate; PE: Phycoerythrin; PI: Propidium iodide; Rev: Reverse; RT-qPCR: Real-time quantitative PCR; siRNA: Small interfering RNA; TK: Tyrosine kinase.}

\section{Competing interests}

The authors declare that they have no competing interests.

\section{Authors' contributions}

ALM and SC performed experiments, statistical analysis, drafted the manuscript and contributed in study conception and intellectual content. DS and MFS participated in acquisition and analysis of the Immunoflurescence experiments. BDR participated in acquisition and analysis of flow cytometry experiments. EA made substantial contributions to the study conception and design and critically revised the manuscript for intellectual content. All authors read and approved the final manuscript.

\section{Acknowledgements}

We thank Dra. Vivian Rumjanek (Departamento de Bioquímica Médica, Universidade Federal do Rio de Janeiro, Brazil) for providing the Lucena cell line and Dr. Pui-Kai Li (Ohio State University, USA) for providing the LLL-3 drug. This work was supported by FINEP, FAPER, CNPQ and Ministério da Saúde (MS).

\section{Author details \\ 'Bone Marrow Transplantation Unit (CEMO), National Cancer Institute (INCA), Rio de Janeiro, Brazil. 'Biophysics and Biometry Department, Roberto Alcântara Gomes Biology Institute, Rio de Janeiro's State University (UERJ), Rio de Janeiro, Brazil. ${ }^{3}$ Department of Obstetrics and Gynecology, University of Ulm, Prittwitzstrasse 43, UIm D-89075, Germany. ${ }^{4}$ Institute of Biophysics Carlos Chagas Filho (IBCCF), Federal University of Rio de Janeiro (UFRJ), Rio de Janeiro, Brazil. ${ }^{5}$ Departamento de Biofísica e Biometria, Instituto de Biologia Roberto Alcântara Gomes, Universidade do Estado do Rio de Janeiro, 28 Avenida de Setembro, 87 Fundos - 4 Andar, Vila Isabel, Rio de Janeiro 20551-030, Brazil.}

Received: 17 September 2014 Accepted: 11 November 2014 Published: 23 November 2014

\section{References}

1. Garcia R, Jove R: Activation of STAT transcription factors in oncogenic tyrosine kinase signalling. J Biomed Sci 1998, 5:79-85.

2. Turkson J, Bowman T, Garcia R, Caldenhoven E, de Groot RP, Jove R: Stat3 activation by Src induces specific gene regulation and is required for cell transformation. Mol Cell Biol 1998, 18:2545-2552.

3. Coppo P, Dusanter-Fourt I, Millot G, Nogueira MM, Dugray A, Bonnet ML, Mitjavila-Garcia MT, Le Pesteur D, Guilhot F, Vainchenker W, Sainteny F, Turhan AG: Constitutive and specific activation of STAT3 by BCR-ABL in embryonic stem cells. Oncogene 2003, 22:4102-4110.

4. Sriuranpong V, Park Jl, Amornphimoltham P, Patel V, Nelkin BD, Gutkind JS: Epidermal growth factor receptor-independent constitutive activation of STAT3 in head and neck squamous cell carcinoma is mediated by the autocrine/paracrine stimulation of the Interleukin 6/gp130 cytokine system. Cancer Res 2003, 63:2948-2956.
5. Coppo P, Flamant S, De Mas V, Jarrier P, Guillier M, Bonnet ML, Lacout C, Guilhot F, Vainchenker W, Turhan AG: BCR-ABL activates STAT3 via JAK end MEK pathways in human cells. Br J Haematol 2006, 134:171-179.

6. Rawlings JS, Rosler KM, Harrison DA: The JAK/STAT signaling pathway. J Cell Sci 2004, 117:1281-1283.

7. Bromberg J, Darnell JE Jr: The role of STATs in transcriptional control and their impact on cellular function. Oncogene 2000, 19:2468-2473.

8. Kiuchi N, Nakajima K, Ichiba M, Fukada T, Narimatsu M, Mizuno K, Hibi M, Hirano T: STAT3 is required for the gp130-mediated full activation of the c-myc gene. J Exp Med 1999, 189:63-73.

9. Hirano T, Ishihara K, Hibi M: Roles of STAT3 in mediating the cell growth, differentiation and survival signals relayed through the IL- 6 family of cytokine receptors. Oncogene 2000, 20:2548-2556.

10. Cheng GZ, Zhang WZ, Sun M, Wang Q, Coppola D, Mansour M, Xu LM, Costanzo C, Cheng JQ, Wang LH: Twist is transcriptionally induced by activation of STAT3 and mediates STAT3 oncogenic function. $J$ Biol Chem 2008, 283:14665-14673.

11. Cambier N, Chopra R, Strasser A, Metcalf D, Elefanty AG: BCR-ABL activates pathways medianting cytokine independence and protection against apoptosis in murine haematopoietic cells in a dose-dependent manner. Oncogene 1998, 16:335-348.

12. Steelman LS, Pohnert SC, Shelton JG, Franklin RA, Bertrand FE, McCubrey JA: JAK/STAT, Raf/MEK/ERK, PI3K/Akt and BCR-ABL in cell cycle progression and leukemogenesis. Leukemia 2004, 18:189-218.

13. Steelman LS, Abrams SL, Whelan J, Bertrand FE, Ludwig DE, Bäsecke J, Libra M, Stivala F, Milella M, Tafuri A, Lunghi P, Bonati A, Martelli AM, McCubrey JA: Contributions of the Raf/MEK/ERK, PI3K/PTEN/Akt/mTOR and Jak/ STAT pathways to leukemia. Leukemia 2008, 22:686-707.

14. McLaughlin J, Chianese $\mathrm{E}$, Witte $\mathrm{ON}$ : In vitro transformation of immature hematopoietic cells by the P210 BCR/ABL oncogene product of the Philadelphia chromosome. Proc Natl Acad Sci U S A 1987, 84:6558-6562.

15. Spiekermann K, Biethahn S, Wilde S, Hiddemann W, Alves F: Constitutive activation of STAT transcription factors in acute myelogenous leukaemia. Eur J Haematol 2001, 67:63-71.

16. Spiekermann K, Pau M, Schwab R, Schmieja K, Franzrahe S, Hiddemann W: Constitutive activation of STAT3 and STAT5 is induced by leukemic fusion proteins with protein tyrosine kinase activity and is sufficient for transformation of hematopoietic precursor cells. Exp Hematol 2002, 30:262-271.

17. Bewry NN, Nair RR, Emmons MF, Boulware D, Pinilla-lbarz J, Hazlehurst LA: STAT3 contributes to resistance toward BCR-ABL inhibitors in a bone marrow microenvironment model of drug resistance. Mol Cancer Ther 2008, 7:3169-3175

18. Chana TK, Lia K, Liua SL, Chua KH, Toha M, Xie WD: Cucurbitacin B inhibits STAT3 and the Raf/MEK/ERK pathway in leukaemia cell line K562. Cancer Lett 2009, 289:46-59.

19. Jia HY, Wu JX, Zhu XF, Chen JM, Yang SP, Yan HJ, Tan L, Zeng YX, Huang W: ZD6474 inhibits Src kinase leading to apoptosis of imatinib-resistant K562 cells. Leuk Res 2009, 33:1512-1519.

20. Espert L, Dusanter-Fourt I, Chelbi-Alix MK: Negative regulation of the JAK/ STAT: pathway implication in tumorigenesis. Bull Cancer 2005, 92:845-857.

21. Yang CH, Shi W, Basu L, Murti A, Constantinescu SN, Blatt L, Croze E, Mullersman JE, Pfeffer LM: Direct association of STAT3 with the IFNAR-1 chain of the human type I interferon receptor. J Biol Chem 1996, 271:8057-8061.

22. Nakayama K, Kim KW, Miyajima A: A novel nuclear zinc finger protein EZI enhances nuclear retention and transactivation of STAT3. EMBO J 2002, 21:6174-6184

23. Sato N, Kawai T, Sugiyama K, Muromoto R, Imoto S, Sekine $Y$, Ishida M, Akira S, Matsuda T: Physical and functional interactions between STAT3 and ZIP kinase. Int Immunol 2005, 17:1543-1552

24. Lufei $C$, Koh TH, Uchida T, Cao X: Pin 1 is required for the Ser727 phosphorylation-dependent Stat3 activity. Oncogene 2007, 26:7656-7664

25. Zhang T, Ma J, Cao X: Grb2 regulates Stat3 activation negatively in epidermal growth factor signalling. Biochem J 2003, 376:457-464.

26. Yamashina K, Yamamoto H, Chayama K, Nakajima K, Kikuchi A: Suppression of STAT3 activity by Duplin, which is a negative regulator of the Wnt signal. J Biochem 2006, 139:305-314.

27. Muromoto R, Nakao K, Watanabe T, Sato N, Sekine Y, Sugiyama K, Oritani K, Shimoda K, Matsuda T: Physical and functional interactions between Daxx and STAT3. Oncogene 2006, 25:2131-2136. 
28. Wu Z, Zhang $X$, Yang J, Wu G, Zhang Y, Yuan Y, Jin C, Chang Z, Wang J, Yang X, He F: Nuclear protein IkappaB-zeta inhibits the activity of STAT3. Biochem Biophys Res Commun 2009, 387:348-352.

29. Collum RG, Brutsaert S, Lee G, Schindler C: A Stat3-interacting protein (StIP1) regulates cytokine signal transduction. Proc Natl Acad Sci 2000, 97:10120-10125.

30. Smith TF, Gaitatzes C, Saxena K, Neer EJ: The WD repeat: a common architecture for diverse functions. Trends Biochem Sci 1999, 24:181-185.

31. Rumjanek VM, Trindade GS, Wagner-Souza K, de-Oliveira MC, MarquesSantos LF, Maia RC, Capella MA: Multidrug resistance in tumour cells: characterization of the multidrug resistant cell line K562-Lucena 1. An Acad Bras Cienc 2001, 73:57-69.

32. Schmittgen TD, Livak KJ: Analyzing real-time PCR data by the comparative C(T) method. Nat Protoc 2008, 3:1101-1108.

33. Nicoletti I, Migliorati G, Pagliaccil MC, Grignani F, Riccardi C: A rapid and simple method for measuring thymocyte apoptosis by propidium iodide staining and flow cytometry. J Immunol Methods 1991, 139:271-279.

34. Benekli M, Baer MR, Baumann H, Wetzler M: Signal transducer and activator of transcription proteins in leukemias. Blood 2003, 15:2940-2954.

35. Mencalha AL, Du Rocher B, Salles D, Binato R, Abdelhay E: LLL-3, a STAT3 inhibitor, represses BCR-ABL-positive cell proliferation, activates apoptosis and improves the effects of Imatinib mesylate. Cancer Chemother Pharmacol 2010, 65:1039-1046.

36. Frank DA: STAT3 as a central mediator of neoplastic cellular transformation. Cancer Lett 2007, 251:199-210.

37. Ichiba M, Nakajima K, Yamanaka Y, Kiuchi N, Hirano T: Autoregulation of the Stat3 gene through cooperation with a cAMP-responsive elementbinding protein. J Biol Chem 1998, 273:6132-6138.

38. Tsuruma R, Ohbayashi N, Kamitani S, Ikeda O, Sato N, Muromoto R, Sekine Y Oritani K, Matsuda T: Physical and functional interactions between STAT3 and KAP1. Oncogene 2008, 27:3054-3059.

39. Corrêa S, Pizzatti L, Du Rocher B, Mencalha A, Pinto D, Abdelhay E: A comparative proteomic study identified LRPPRC and MCM7 as putative actors in imatinib mesylate cross-resistance in Lucena cell line. Proteome Sci 2012, 10:23.

40. Hawkes NA, Otero G, Winkler GS, Marshall N, Dahmus ME, Krappmann D, Scheidereit C, Thomas CL, Schiavo G, Erdjument-Bromage H, Tempst P, Svejstrup JQ: Purification and characterization of the human elongator complex. J Biol Chem 2002, 277:3047-3055.

41. Ma LD, Zhou M, Wen SH, Ni C, Jiang LJ, Fan J, Xia L: Effects of STAT3 silencing on fate of chronic myelogenous leukemia K562 cells. Leuk Lymphoma 2010, 51:1326-1336.

42. Bourguignon LYW, Peyrollier K, Xia W, Gilad E: Hyaluronan-CD44 interaction activates stem cell marker Nanog, Stat-3-mediated MDR1 gene expression, and ankyrin-regulated multidrug efflux in breast and ovarian tumor cells. J Biol Chem 2008, 283:17635-1751.

43. Zhang X, Xiao W, Wang L, Tian Z, Zhang J: Deactivation of signal transducer and activator of transcription 3 reverses chemotherapeutics resistance of leukemia cells via down-regulating P-gp. PLoS One 2011, 6:20965-20969.

doi:10.1186/1471-2407-14-866

Cite this article as: Mencalha et al: Inhibition of STAT3-interacting protein 1 (STATIP1) promotes STAT3 transcriptional up-regulation and imatinib mesylate resistance in the chronic myeloid leukemia. BMC Cancer 2014 14:866.

\section{Submit your next manuscript to BioMed Central and take full advantage of:}

- Convenient online submission

- Thorough peer review

- No space constraints or color figure charges

- Immediate publication on acceptance

- Inclusion in PubMed, CAS, Scopus and Google Scholar

- Research which is freely available for redistribution 